\title{
SCALING OF WAKEFIELD EFFECTS IN RECIRCULATING LINACS
}

\author{
L. Merminga ${ }^{\dagger}$, G. R. Neil, B. C. Yunn, Jefferson Laboratory, Newport News, VA 23606 \\ J. J. Bisognano, Synchrotron Radiation Center, Stoughton, WI 53589
}

\begin{abstract}
Expressions for the induced energy spread and emittance degradation of a single bunch due to the longitudinal and transverse impedance of rf cavities at the end of a linac structure are presented. Scaling of the formulae with $\mathrm{rf}$ frequency is derived. Scaling of the threshold current for the multibunch, multipass beam breakup (BBU) instability in recirculating linacs with accelerator and beam parameters is also derived.
\end{abstract}

\section{INTRODUCTION}

Recirculating, energy recovering linacs (ERLs) can be used as efficient driver accelerators for high average power Free Electron Lasers, synchrotron radiation light sources and colliders. The efficiency of ERLs increases as the beam intensity increases. Furthermore, for all these applications the beam at the end of the acceleration must maintain small energy spread and small transverse size in spite of strong wakefields. In this note we examine the scaling of wakefield effects with accelerator and beam parameters and, in particular, how the choice of $\mathrm{rf}$ frequency influences the wakefield effects.

\section{MULTIBUNCH - MULTIPASS BEAM BREAKUP}

For a single cavity, a single higher order mode (HOM) and a single recirculation, the multipass, multibunch beam breakup threshold current can be approximated by [1],

$$
I_{t h}=\frac{-2 p_{r} c}{e(R / Q)_{m} Q_{m} k_{m} M_{12}^{r}}
$$

where the most pessimistic conspiracy of time delay has been assumed. Here $p_{r}$ is the momentum of the recirculating beam, $(R / Q)_{m}$ is the shunt impedance of the structure for the particular HOM, in units of Ohms, $k_{m}=\omega_{n} / c$ is the wavenumber of the HOM and $M_{12}$ is the transfer matrix element for the recirculation path in the $\left(\mathrm{x}, \mathrm{x}^{\prime}\right)$ space.

To investigate the scaling of the BBU threshold current with rf frequency, we consider structures which provide the same energy gain at different $r f$ frequencies. Next we explore the scaling under two different assumptions: a) gradient is frequency-independent and b) gradient varies linearly with frequency.

\subsection{Gradient is Frequency-Independent}

If the gradient is frequency-independent, then in order

merminga@jlab.org to have the same energy gain, the structures must have the same length at all frequencies. Then the shunt impedance of the structure $(R / Q)_{m}$ is equal to $(r / Q)_{m} L \sim \omega L$, where $(r / Q)_{m}$ is the shunt impedance per unit length and scales linearly with frequency [2].

Now we discuss the scaling of $Q_{m}$ with $\mathrm{rf}$ frequency. The value of the external $Q$ of the HOM depends on the coupling of the mode and it is, in principle, independent of frequency. Lower frequencies however tend to favor lower $Q$ values for two reasons: first, because at lower frequencies there are typically fewer cells per structure, which makes coupling of HOMs easier; second, there are fewer trapped modes in a structure with fewer cells. Because of these considerations, $Q_{m}$ will be left out explicitly in the scaling. Finally $p_{r}$ and $M_{12}$ have no explicit frequency dependence and $k_{m} \sim \omega$.

In summary, if the gradient is assumed frequencyindependent then the threshold current scales with the rf frequency as

$$
I_{t h} \sim \frac{1}{\omega^{2} Q_{m}}
$$

\subsection{Gradient is a Linear Function of Frequency}

If the gradient varies linearly with frequency, then as one scales to a different frequency, the length of the structure must also scale accordingly in order to maintain the same energy gain. Specifically, if $E_{a c c} \sim \omega$, then $L \sim 1 / \omega$ for constant energy gain. Therefore

$$
(R / Q)_{m}=(r / Q)_{m} L \sim \omega \frac{1}{\omega} \sim \text { constant }
$$

and the threshold current scales as

$$
I_{t h} \sim \frac{1}{\omega Q_{m}} .
$$

In general, if $L_{a c c}$ is the total length of the accelerator, then the multibunch BBU threshold current varies as,

$$
I_{t h} \sim \frac{1}{\omega^{2} L_{a c c} Q_{m}}
$$

independently of the gradient scaling with rf frequency.

\section{LONGITUDINAL IMPEDANCE}

The induced energy spread due to longitudinal impedances is given by

$$
\Delta E \simeq 2 k_{\|} Q
$$

where $Q$ is the charge per bunch and $k_{\|}$is the loss factor due to longitudinal wakes. For a single-cell rf cavity the 
longitudinal loss factor is given to a good approximation by [3],

$$
k_{\|}^{\text {cavity }}=\frac{Z_{0} c}{2 \pi^{2} a} \sqrt{\frac{g}{\sigma}}
$$

where $g$ is the gap of the cavity, $\sigma$ is the rms bunch length, $a$ is the beam pipe radius and $Z_{0}=377 \Omega$ is the impedance of free space. If there are $N_{\text {cell/cav }}$ cells per cavity of length $l$ and if they are close enough together so that $N_{\text {eff }}>>1$, where $N_{\text {eff }}$ is defined by

$$
N_{\text {eff }} \simeq \frac{k a^{2}}{l} \simeq \frac{1}{\pi} \frac{a^{2}}{\sigma l}
$$

and expresses the number of cells which interfere with each other, then the total induced energy spread does not increase linearly with $N_{\text {cell/cav }}$ but instead it increases as the $\sqrt{ } N_{\text {cell/cav }}$. This is true until the number of cells per cavity is approximately equal to $N_{\text {eff: }}$ For $N_{\text {cell/cav }} \geq N_{\text {eff }}$ the contribution from the individual cells start to add again, until, in the asymptotic regime, one finds the expression for the loss factor of an infinite periodic structure, given by

$$
k_{\|}^{\infty \text { periodic }}=\frac{Z_{0} c}{2 \pi} \frac{1}{a^{2}} l .
$$

Therefore, the induced energy spread due to the longitudinal impedance of $N_{c a v}$ cavities, is given by

$$
\Delta E \simeq N_{\text {cav }} \sqrt{N_{\text {cell /cav }}} 2 k_{\|}^{\text {cavity }} Q
$$

as long as $N_{\text {cell/cav }}$ remains less than or approximately equal to $N_{\text {eff }}$

\subsection{Scaling of Longitudinal Impedance}

We assume the most straightforward scaling of the physical dimensions of a cell with frequency, namely

$$
g, a \sim \frac{1}{\omega} .
$$

We also assume that the bunch length scales inversely proportional to frequency, $\sigma \sim 1 / \omega$. If additional bunching is needed at the final beam energy, such as the case might be in an FEL, this can take place at the end of the acceleration section with the use of magnetic elements. Under these assumptions the energy spread scales with rf frequency as

$$
\Delta E \sim N_{\text {cav }} \sqrt{N_{\text {cell/cav }}} \omega Q
$$

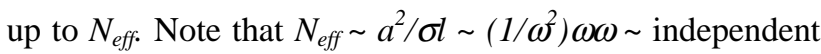
of frequency. Namely, the number of cells over which there is interference is the same at all frequencies, and the multiplicative factor involving the number of cells is the same for all frequencies.

\section{TRANSVERSE IMPEDANCE}

The emittance degradation due to transverse wakefields can be expressed as

$$
r_{e}=1+\frac{x_{0} \eta}{2 \pi} \sqrt{\frac{\gamma_{i}}{\beta \varepsilon_{n}}}
$$

for small $\eta$, where $\eta$ is given by [4],

$$
\eta \simeq \frac{6 Q\left(k_{\perp} / l\right) L_{\text {accelerator }}}{\mathrm{k}_{\beta}\left(E_{f}-E_{i}\right)} \ln \left(1+\frac{E_{f}-E_{i}}{E_{i}}\right)
$$

where $\left(k_{\perp} / l\right)$ is the total impedance of the whole accelerator per unit length, $x_{0}$ is the beam offset, $\beta$ is the betatron function and $\varepsilon_{n}$ is the normalized emittance. This expression assumes no emittance compensation schemes, such a BNS damping. For a single cavity, the transverse loss factor is given to a good approximation by,

$$
k_{\perp}^{\text {cavity }}=\frac{Z_{0} c}{4 \pi} \frac{1}{a^{3}} \sqrt{\pi g \sigma} .
$$

For $N_{\text {cell/cav }}$ cells per cavity, up to $N_{\text {eff, }}$,

$$
\eta \approx \frac{1}{\sqrt{N_{\text {cell/cav }}}} \frac{6 Q}{a^{3}} \sqrt{\frac{\pi \sigma}{g}} L_{a c c}
$$

where now the single-cell loss factor is multiplied by the square root of the number of cells to account for the interference between cells, but also the length becomes $N_{\text {cell/cav }}$ times longer. Just as in the longitudinal case, the transverse impedance for an infinite periodic structure of length $l$ can be derived by adding up linearly the contributions of each cell beyond the $\sim N_{\text {eff }}$ th one, to arrive at

$$
k_{\perp}^{\infty \text { periodic }} \simeq \frac{Z_{0} c}{4 \pi} \frac{1}{a^{4}} \sigma l
$$

\subsection{Scaling of Transverse Impedance}

Assuming that all cavity dimensions and the bunch length vary inversely proportionally to the rf frequency, $a, g, l, \sigma \sim 1 / \omega$, we arrive at

$$
\eta \sim \frac{L_{a c c}}{\sqrt{N_{\text {cell/cav }}}} Q \omega^{3}
$$

up to $N_{\text {eff }}$.

\section{ACKNOWLEDGEMENTS}

This work was supported by the USA DoE contract No DE-AC05-84-ER40150, the Office of Naval Research, The Commonwealth of Virginia, and the Laser Processing Consortium.

\section{REFERENCES}

[1] G. Krafft, J. Bisognano and S. Laubach, "Beam Breakup in Superconducting Linear Accelerators," Jefferson Lab Technical Report (1988)

[2] P. Wilson, "High Energy Electron Linacs: Applications to Storage Ring RF Systems and Linear Colliders," SLAC-PUB-2882 (1982) 
[3] J. Bisognano, S. A. Heifets, B. C. Yunn, "Impedances of CEBAF Superconducting Accelerator," Jefferson Lab Technical Report TN-0059 (1988)

[4] A. Chao, B. Richter, C.-Y. Yao, "Beam Emittance Growth Caused by Transverse Deflecting Fields in a Linear Accelerator," NIM A 178:1-8 (1980) 